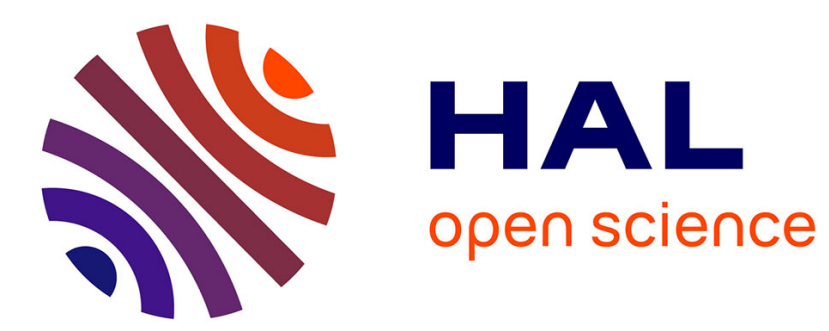

\title{
Workload analysis of a two-queue system by formulating a boundary value problem \\ Philippe Nain
}

\section{To cite this version:}

Philippe Nain. Workload analysis of a two-queue system by formulating a boundary value problem. RR-0190, INRIA. 1983. inria-00076368

\section{HAL Id: inria-00076368 \\ https://hal.inria.fr/inria-00076368}

Submitted on 24 May 2006

HAL is a multi-disciplinary open access archive for the deposit and dissemination of scientific research documents, whether they are published or not. The documents may come from teaching and research institutions in France or abroad, or from public or private research centers.
L'archive ouverte pluridisciplinaire HAL, est destinée au dépôt et à la diffusion de documents scientifiques de niveau recherche, publiés ou non, émanant des établissements d'enseignement et de recherche français ou étrangers, des laboratoires publics ou privés. 


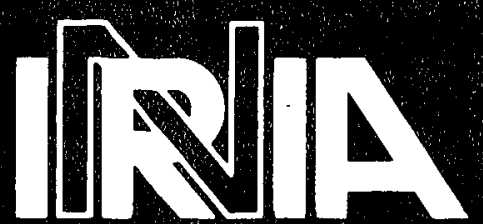

6

CENTRE DE ROCOUENCOURT

Institut National de Recherche en Informatique et en Automatique

Domaine de Voluceau Rocowencourt $\mathrm{BP} 105$ 78153 Le Crinay Cedex ranee Tél:.9549020

\section{Rapports de Recherche}

$$
\mathrm{N}^{\circ} 190
$$

\section{WORKLOAD ANALYSIS OF A TWO-QUEUE SYSTEM BY FORMULATING A BOUNDARY VALUE PROBLEM}




\title{
WORKLOAD ANALYSIS OF A TWO-QUEUE SYSTEM BY FORMULATING A BOUNDARY VALUE PROBLEM
}

\author{
Philippe NAIN \\ INRIA \\ Bomaine de Voluceau \\ Rocquencourt \\ 78150 Le Chesnay
}

\section{RESUME}

Nous étudions un nouveau protocole de communication qui réalise 1 'insertion de messages spéciaux dans un flot de messages réguliers. L'analyse se ramène à 1 'étude de deux files d'attente et d'un serveur unique. La discipline de service dépend de la charge d'une des files. Nous calculons, à I'état stationnaire, les transformées de Fourier Stieltjes et Laplace - Stieltjes de la distribution de la charge du système, en résolvant deux équations fonctionnelles.

\section{ABSTRACT}

We analyse a new communication protocol which regulates the merging of special messages in a regular flow. The study is carried out via a queueing model consisting of two waiting lines and one single server facility. The server sharing policy depends on the workload of one of the waiting lines. We derive the Fourier - Stieltjes and Laplace - Stieltjes transforms of the joint stationary distribution of the system-workload by solving two functional equations.

Keywords : Markov process ; Functional equation ; Wiener-Hopf factorization ; Algebraic curve ; Dirichlet problem. 


\section{INTRODUCTION}

Queueing models with state-dependency, connected to the coupling of processors in computer systems, have been extensively studied recent1y. Analytic methods have been developed by Fayolle and Iasnogorodski allowing the solution of various coupling problems [FAY 79], [IAS 79], [FAY, IAS 79] and leading to a fairly general methodology in this field [BOX, COH 81], [MIK 81], [BAC, FAY 82], [FAY, KIN, MIT 82], [BLA 82].

The approach involves the solution of functional equations. Genera1ly the unknown functions are the generating functions of the joint stationary distribution of the number of jobs in the system. This, in turn, leads to the resolution of boundary value problems (Dirichlet and Riemann-Hilbert problems).

We use a similar machinery to study a new communication protocol which regulates the insertion of special (priority) messages in a regular flow.

The analysis is carried out via a queueing model consisting of two queues and one single server. The communication protocol can be described as follows : the customers in the special messages queue are served when the workload - the amount of required service times - in the regular messages queue remains below a given threshold. Section I gives a more carefull description of the model.

The Fourier - Stieltjes and Laplace - Stieltjes transforms of the joint stationary distribution of the system - workload are obtained, by solving an "exterior" boundary value problem on a circle.

In Section 2, we get a functional equation satisfied by the workload of the system.

Then, an intermediate Wiener-Hopf factorization [Section 3] allows the reduction to an exterior boundary value problem on a circle, which. can be solved. Closed forms are obtained [Section 4].

The results are summarized in a theorem. Figures are given, showing the effect of this server sharing policy upon the mean workload in each queue. 
1. - THE MODEL

We consider the following queueing model :

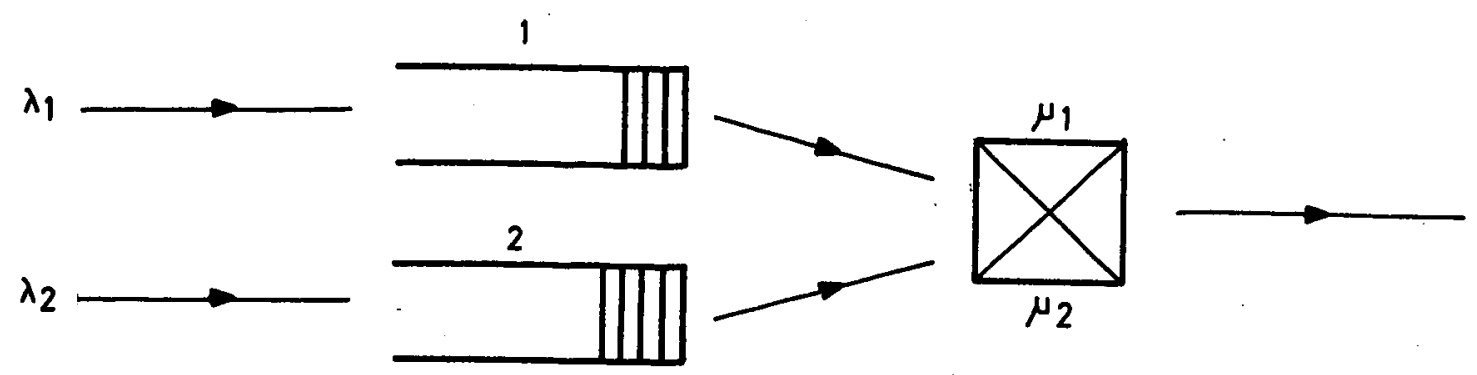

The arrivals form two independent Poisson processes with parameters $\lambda_{1}, \lambda_{2}$

The service times of the customers of queue $j \stackrel{\text { def }}{=}$ customers of type $j$ ) form a renewal process with an absolutely continuous but otherwise arbitrary renewal distribution function and with mean $1 / \mu_{j}(j=1,2)$. A11 the processes are assumed to be mutually independent.

The service discipline is first-in-first-out in each queue. The server sharing policy between the two types of customers is the following : when the occupation time of the server - the workload - w.r.t. the customers of type 1 is greater than $c$ [resp. smaller or equal to $c$ ] (c is fixed, $c \in \mathbf{R}^{+}$) a customer of type 1 [resp. type 2] is served. Moreover, customers of each type can be preempted and they will resume their service demand ("preemptive resume priority").

For $j=1,2$, we define :

- $\beta_{j}(s)$ the Laplace-Stieltjes transform of the service times distribution of customers of type $j$, for $\operatorname{Re} s \geq 0$. Re $s$ denotes the real part of the complex number $s$.

$-v_{j}(t)$ the workload of queue $j$ at time $t(t>0)$. 


\section{2. - THE FUNGTIONAL EQUATION}

The stochastic process. $v \stackrel{\operatorname{def}}{=}\left\{\mathrm{V}_{1}(t), \mathrm{V}_{2}(t), t>0\right\}$ is a Markov process.

Let $v_{\Delta t}^{j}$ be the number of arrivals in queue $j$ in $(t, t+\Delta t], \tau_{j}$ be the service time required by the arriving customer in queue $j$ in $(t, t+\Delta t]$, $(j=1,2)$ and let us define

$H(x, y ; t) \stackrel{\operatorname{def}}{=} E\left\{e^{-x V_{1}(t)-y V_{2}(t)}\right\}$ for $\operatorname{Re} x \geq 0$, Re $y \geq 0, t>0$

The Kolmogorov forward equations for the $V$-process are for

$$
\begin{array}{rlrl}
v_{\Delta t}^{1}=v_{\Delta t}^{2}=0, & (t>0): \\
v_{1}(t+\Delta t) & =\left[v_{1}(t)-\Delta t\right]^{+} & \text {if }\left\{v_{1}(t)>c\right\} \text { or }\left\{v_{1}(t) \leq c, v_{2}(t)=0\right\} \\
& =v_{1}(t) & & \text { if }\left\{v_{1}(t) \leq c, v_{2}(t)>0\right\} \\
v_{2}(t+\Delta t) & =v_{2}(t) & & \text { if }\left\{v_{1}(t)>c\right\} \text { or }\left\{v_{1}(t) \leq c, v_{2}(t)=0\right\} \\
& =v_{2}(t)-\Delta t & & \text { if }\left\{v_{1}(t) \leq c, v_{2}(t)>0\right\}
\end{array}
$$

For $v_{\Delta t}^{1}=0, v_{\Delta t}^{2}=1$, we have :

$$
\begin{aligned}
V_{1}(t+\Delta t) & =v_{1}(t)-\Delta t & & \text { if }\left\{V_{1}(t)>c\right\} \\
& =\left[V_{1}(t)-\varepsilon \Delta t\right]^{+} & & \text {if }\left\{V_{1}(t) \leq c, V_{2}(t)=0\right\} \\
& =V_{1}(t) & & \text { if }\left\{V_{1}(t) \leq c, V_{2}(t)>0\right\}
\end{aligned}
$$

$$
\begin{array}{rlrl}
v_{2}(t+\Delta t) & =V_{2}(t)+\tau_{2} & & \text { if }\left\{v_{1}(t)>c\right\} \\
& =\tau_{2}-(1-\varepsilon) \Delta t \quad \text { if }\left\{v_{1}(t) \leq c, v_{2}(t)=0\right\} \\
& =v_{2}(t)-\Delta t+\tau_{2} \text { if }\left\{v_{1}(t) \leq c, v_{2}(t)>0\right\}
\end{array}
$$




$$
\begin{aligned}
& \text { For } v_{\Delta t}^{1}= 1, v_{\Delta t}^{2}=0 \text {, we have : } \\
& \begin{aligned}
v_{1}(t+\Delta t) & =v_{1}(t)-\Delta t+\tau_{1} \text { if }\left\{v_{1}(t)>c\right\} \text { or }\left\{0<v_{1}(t) \leq c, v_{2}(t)=0\right\} \\
& =v_{1}(t)-\varepsilon^{\prime} \Delta t+\tau_{1} \text { if }\left\{v_{1}(t)+\tau_{1}>c, v_{1}(t) \leq c, v_{2}(t)>0\right\} \\
& =v_{1}(t)+\tau_{1} \quad \text { if }\left\{v_{1}(t)+\tau_{1} \leq c, v_{1}(t) \leq c, v_{2}(t)>0\right\} \\
& =\tau_{1}-\varepsilon^{\prime} \Delta t \quad \text { if }\left\{v_{1}(t)=v_{2}(t)=0\right\} \\
& \\
v_{2}(t+\Delta t) & =v_{2}(t) \quad \text { if }\left\{v_{1}(t)>c\right\} \text { or }\left\{v_{1}(t) \leq c, v_{2}(t)=0\right\} \\
& =v_{2}(t)-\Delta t \quad \text { if }\left\{v_{1}(t)+\tau_{1} \leq c, v_{1}(t) \leq c, v_{2}(t)>0\right\} \\
& =v_{2}(t)-\left(1-\varepsilon^{\prime}\right) \Delta t \text { if }\left\{v_{1}(t)+\tau_{1}>c, v_{1}(t) \leq c, v_{2}(t)>0\right\}
\end{aligned}
\end{aligned}
$$

where $\varepsilon$ and $\varepsilon^{\prime}$ are two independent random variables taking their values in $] 0,1[$.

From the above relations and the Poissonian arrival assumptions, it readily follows that for $\operatorname{Re} x \geq 0$, Re $y \geq 0$ :

$$
\begin{aligned}
& E\left\{e^{-x V_{1}(t+\Delta t)-y V_{2}(t+\Delta t)} / \nu_{\Delta t}^{1}=v_{\Delta t}^{2}=0\right\} P\left(\nu_{\Delta t}^{1}=v_{\Delta t}^{2}=0\right)=H(x, y ; t) \\
& +(y-x) E\left\{e^{-x V_{1}(t)-y V_{2}(t)}\left(v_{1}(t) \leq c, v_{2}(t)>0\right)\right\} \\
& -x P\left(V_{1}(t)=V_{2}(t)=0\right)+o(\Delta t)
\end{aligned}
$$

and :

$$
\begin{aligned}
E\left\{e^{-x V_{1}(t+\Delta t)-y V_{2}(t+\Delta t)} /\left(\nu_{\Delta t}^{1}, \nu_{\Delta t}^{2}\right)\right. & =(k, l)\} P\left(\left(v_{\Delta t}^{1}, v_{\Delta t}^{2}\right)=(k, l)\right) \\
& =\lambda_{m} \beta_{m}\left(s_{m}\right) H(x, y ; t)+o(\Delta t)
\end{aligned}
$$

where $(k, l) \in\{(0,1),(1,0)\}$ and where $m=1$ if $k=1$ and $m=2$ if $k=0$. 
Summing these three relations, dividing by $\Delta t$ and letting $\Delta t \downarrow 0$, we obtain the following time dependent functional equation for the workload :

$$
\begin{aligned}
\frac{\partial}{\partial t} H(x, y ; t) & =\left(x-\lambda_{1} \gamma_{1}(x)-\lambda_{2} \dot{\gamma}_{2}(y)\right) H(x, y ; t) \\
& +(y-x) E\left\{e^{-x V_{1}(t)-y V_{2}(t)}\left(V_{1}(t) \leq c, V_{2}(t)>0\right)\right\} \\
& -x P\left(V_{1}(t)=V_{2}(t)=0\right)
\end{aligned}
$$

where $\gamma_{j}(s) \stackrel{\text { def }}{=} 1-\beta_{j}(s)$ for $\operatorname{Re} s \geq 0$ and $j=1,2$.

The queueing system investigated has the "workload conservation" próperty, because of the "resume" priority. Consequently, the probability distribution of the duration of a busy period of the whole system, is the same, for instance, as the one of the same queueing model with the classical preemptive resume priority.

Thus these two models have the same ergodicity condition. But we know the ergodicity condition of the queueing system with the classical preemptive resume priority, which is :

$$
\rho_{1}+\rho_{2}<1
$$

with $\rho_{j} \stackrel{\text { def }}{=} \lambda_{j} / \mu_{j}(j=1,2)[\operatorname{COH} 69]$. In the following, we assume that this condition holds.

Under this assumption, the Markov process $v=\left\{v_{1}(t), v_{2}(t), t>0\right\}$ possesses a unique stationary distribution.

Let $v_{1}, v_{2}$ be two random variables with distribution this stationary

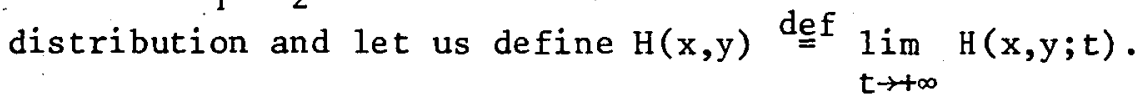

From equation ( 1 ) we then obtain for $\operatorname{Re} x \geq 0$, Re $y \geq 0$, the following basic functional equation characterizing the workload of the system at steady state : 


$$
\begin{aligned}
\left(x-\lambda_{1} \gamma_{1}(x)-\lambda_{2} \gamma_{2}(y)\right) H(x, y) & =(x-y) E\left\{e^{-x V_{1}-y V_{2}}\left(V_{1} \leq c, V_{2}>0\right)\right\} \\
& +x E\left\{\left(V_{1}=V_{2}=0\right)\right\}
\end{aligned}
$$

\section{Some remarks concerning equation (2):}

Taking $y=0$, dividing by $x$ and letting $x+0$ in (2), leads to :

$$
1-\rho_{1}=E\left\{\left(V_{1} \leq c, V_{2}>0\right)\right\}+P\left(V_{1}=V_{2}=0\right)
$$

In'the same way, taking $\mathrm{x}=0$, dividing by $\mathrm{y}$ and letting $\mathrm{y}+0$, leads to:

$$
\rho_{2}=P\left(V_{1} \leq c, V_{2}>0\right)
$$

From relations (3) and (4), we deduce the expected result :

$$
P\left(V_{1}=V_{2}=0\right)=1-\rho_{1}-\rho_{2}
$$

- If $c=0$, the service discipline is the classical preemptive resume priority, where the customers of type 1 Kold the higher degree of priority. In this case and for $y=0$ equation (2) becomes the well known TAKÁCS equation, stating the workload in a $M / G / 1$ queueing system at steady state [TAK 52].

- If $c \rightarrow+\infty$, the same comments to the ones above can be made, changing "type 1" for "type 2" and "y = 0" for " $\mathrm{x}=0$ ".

In what follows, we shall assume that $0<c<+\infty$ and that the service times of customers of type 1 are exponentially distributed, with mean $1 / \mu_{1}$ (this implies $\gamma_{1}(s)=\frac{s}{\mu_{1}+s}$ ).

\section{3. - THE WIENER-HOPF DECOMPOSITION}

We obtain, in this section, a Wiener-Hopf decomposition of the function $E\left\{e^{-x V_{1}-y V_{2}}\right\}$ in order to solve (sections 4 and 5 ) equation (2) for $\operatorname{Re} x=0, \operatorname{Re} y \geq 0[\mathrm{COH} 79]$. Set : 


$$
\begin{aligned}
& \mathrm{H}^{-}(\mathrm{x}, \mathrm{y}) \stackrel{\text { def }}{=} E\left\{e^{-\mathrm{x}\left(\mathrm{V}_{1}-\mathrm{c}\right)-\mathrm{y} \mathrm{V}_{2}}\left(\mathrm{~V}_{1} \leq \mathrm{c}\right)\right\} \\
& \mathrm{H}^{+}(\mathrm{x}, \mathrm{y}) \stackrel{\text { def }}{=} E\left\{e^{-x\left(V_{1}-c\right)-y v_{2}}\left(\mathrm{~V}_{1}>c\right)\right\}
\end{aligned}
$$

$\mathrm{H}^{-}(\mathrm{x}, \mathrm{y})\left[\operatorname{resp~} \mathrm{H}^{+}(\mathrm{x}, \mathrm{y})\right]$ is analytic in $\mathrm{x}$ for $\operatorname{Re} \mathrm{x}<0$ [resp. Re $\mathrm{x}>0$ ] and is continuous in $x$ for $\operatorname{Re} x \leq 0$ [resp. Re $x \geq 0]$.

$$
\begin{aligned}
& \text { For } \operatorname{Re} x=0, \operatorname{Re} y \geq 0 \text {, it follows : } \\
& H(x, y)=e^{-x c}\left(H^{-}(x, y)+H^{+}(x, y)\right) \\
& \text { Let us define : } \\
& \frac{P^{-}(x, y)}{\mu_{1}+x} \stackrel{\text { def }}{=}\left(y-\lambda_{1} \gamma_{1}(x)-\lambda_{2} \gamma_{2}(y)\right) H^{-}(x, y)-x\left(1-\rho_{1}-\rho_{2}\right) e^{x c} \\
& +(x-y) E\left\{e^{-x\left(v_{1}-c\right)}\left(v_{1}<c ; v_{2}=0\right)\right\} \\
& \frac{P^{+}(x, y)}{\mu_{1}+x} \stackrel{\text { def }}{=}-\left(x-\lambda_{1} \gamma_{1}(x)-\lambda_{2} \gamma_{2}(y)\right) H^{+}(x, y)
\end{aligned}
$$

Hence from (6), it is seen that $\mathrm{P}^{-}\left[\right.$resp. $\left.\mathrm{P}^{+}\right]$has the same property that $\mathrm{H}^{-}$[resp. $\left.\mathrm{H}^{+}\right]$concerning his analycity and continuity domains in the variable $x$.

$$
\begin{aligned}
& \text { Moreover, for } \operatorname{Re} \mathrm{x}=0, \operatorname{Re} \mathrm{y} \geq 0 \text {, we have from (2) and (6): } \\
& \mathrm{P}^{-}(\mathrm{x}, \mathrm{y})=\mathrm{P}^{+}(\mathrm{x}, \mathrm{y})
\end{aligned}
$$

On the other hand, $\left|P^{-}(x, y)\right|\left[\right.$ resp. $\left.\left|P^{+}(x, y)\right|\right]$ is $O\left(\left|x^{2}\right|\right)$ where $|z|$ denotes the modulus of the complex number $z$. Applying Liouville's theorem it is seen that $P$ defined by :

* we obvious $1 y$ have $P\left(V_{1}=c, V_{2}=0\right)=0$ since we assumed $c \neq 0$ 


$$
P(x, y) \stackrel{\operatorname{def}}{=}\left\{\begin{array}{ll}
P^{-}(x, y) & \text { if } \operatorname{Re} x \leq 0 \\
P^{+}(x, y) & \text { if } \operatorname{Re} x \geq 0
\end{array} \quad \text { for Re } y \geq 0\right.
$$

and which is therefore analytic in $x$, is a polynomial of degree at most two in $x$. Dividing $P$ by $x$ and letting $|x|++\infty$ with for instance Re $x>0$, it is readily seen that the coefficient of $x^{2}$ must be zero.

For $\operatorname{Re} y \geq 0$, we then obtain the following system :

(7) $\begin{cases}R(x, y) H^{-}(x, y)=f(x)+g(x, y) A(x)+\frac{x B(y)+C(y)}{\mu_{1}+x} & \text { for } \operatorname{Re} x \leq 0 \\ S(x, y) H^{+}(x, y)=-\frac{x B(y)+C(y)}{\mu_{1}+x} & \text { for } \operatorname{Re} x \geq 0\end{cases}$

where :

$$
\begin{aligned}
& A(x) \stackrel{\operatorname{def}}{=} E\left\{e^{-x\left(V_{1}-c\right)}\left(V_{1}<c, v_{2}=0\right)\right\} \\
& B(y), C(y) \text { are the unknown coefficients in } y \text { of the polynomial } \\
& \text { in } x, P(x, y) \\
& R(x, y) \stackrel{\text { def }}{=}\left(y-\lambda_{1} \gamma_{1}(x)-\lambda_{2} \gamma_{2}(y)\right) \\
& S(x, y) \stackrel{\text { def }}{=}\left(x-\lambda_{1} \gamma_{1}(x)-\lambda_{2} \gamma_{2}(y)\right) \\
& f(x) \stackrel{\text { def }}{=} x\left(1-\rho_{1}-\rho_{2}\right) e^{c x} \\
& g(x, y) \stackrel{\text { def }}{=} y-x .
\end{aligned}
$$

A central role in the analysis is played by the "kernel" $R(x, y)$ [resp. $S(x, y)]$ because if for a pair $(x, y)$, Re $x \leq 0$ [resp. Re $x \geq 0$, $\operatorname{Re} y \geq 0$, this kernel vanishes, then the righthand side of equation (7.1) [resp. (7.2)] must also vanish.

Using this property, we see that it is easy to find a relation between the two unknown coefficients $B(y)$ and $C(y)$, from equation (7.2). 
For a fixed $y$, Re $y \geq 0$, the equation $S(x, y)=0$ possesses a unique root $x=Z(y)$ in the half-plane $\operatorname{Re} x \geq 0$ ([COH 69], p. 536).

Moreover, $\mathrm{Z}(\mathrm{y})$ is an analytic function for $\operatorname{Re} \mathrm{y}>0$, and is given by :

$$
Z(y)=\lambda_{2} \gamma_{2}(y)+\lambda_{1}\left(1-E\left\{e^{-\lambda_{2} \gamma_{2}(y) P}\right\}\right)
$$

where $P$ stands for the busy period of an $M / G / 1$ queueing system with average arrival rate $\lambda_{1}$ and Laplace-Stieltjes transform of the service times distribution $\beta_{1}(s)$, Re $s \geq 0$ ([ $[\mathrm{COH} 69]$, p. 536). (With this method, it is easy from eq. 2 to determine $\mathrm{H}(\mathrm{x}, \mathrm{y})$ for $\mathrm{c}=0$ or $\mathrm{c}++\infty)$.

We then have :

$C(y)=-Z(y) \quad B(y) \quad \forall \operatorname{Re} y \geq 0$

The first equation (7.1) of system (7) becomes for $\operatorname{Re} x \leq 0, \operatorname{Re} y \geq 0$ :

$$
R(x, y) \cdot H^{-}(x, y)=f(x)+g(x, y) A(x)+h(x, y) B(y)
$$

where :

$$
h(x ; y) \stackrel{\text { def }}{=} \frac{x-Z(y)}{\mu_{1}+x}
$$

In the seque1, we examine carefully the algebraic curve defined by $T(x, y) \stackrel{\text { def }}{=}\left(\mu_{1}+x\right)\left(\mu_{2}+y\right) R(x, y)=0$ in the whole complex plane.

\section{$\underline{\text { Remarks : }}$}

- Taking $x=0$. in (7.2), we see that $C(y)$ is analytic for $\operatorname{Re} y>0$, since $\gamma_{2}(y)$ and $H^{+}(0, y)$ are analytic for $\operatorname{Re} y>0$.

Then, from (10), we deduce that $\mathrm{B}(\mathrm{y})$ must be analytic for $\operatorname{Re} \mathrm{y}>0$ $(z(y)=0$ for $\operatorname{Re} y \geq 0$ iff $y=0)$.

- If $\beta_{j}(s)=\frac{\mu_{j}}{\mu_{j}+s}$, Re $s \geq 0, j=1,2$, we have : 
$z(y)=\frac{\lambda_{2} y+\left(\mu_{2}+y\right)\left(\lambda_{1}-\mu_{1}\right)+\sqrt{\left(\left(\lambda_{1}+\mu_{1}\right)\left(\mu_{2}+y\right)+\lambda_{2} y\right)^{2}-4 \lambda_{1} \mu_{1}\left(\mu_{2}+y\right)^{2}}}{2\left(\mu_{2}+y\right)}$

for $\operatorname{Re} y \geq 0$ (cf. [KLE 75], p. 215).

From now on, we sha11 assume that $\beta_{j}(s)=\frac{\mu_{j}}{\mu_{j}+s}, j=1,2$ (the service times distribution of customers of type $j$ are exponentially distributed, with mean $\left.1 / \mu_{j}, j=1,2\right)$.

\section{4. - DETERMINATION OF A AND B}

We assume $\lambda_{1} \neq 0$ and $\lambda_{2} \neq 0$.

$$
\text { 4.1. }-\underline{T(x, y)=0}
$$

Solving $T(x, y)=0$ in $y, x$ being fixed, $x \in C$, we obtain the algebraic function :

$$
Y(x) \operatorname{def} \frac{\lambda_{1} x-\left(\mu_{2}-\lambda_{2}\right)\left(\mu_{1}+x\right) \pm \sqrt{\Delta(x)}}{2\left(\mu_{1}+x\right)}
$$

where :

$$
\Delta(x) \stackrel{\text { def }}{=}\left(\lambda_{1} x-\left(\mu_{2}-\lambda_{2}\right)\left(\mu_{1}+x\right)\right)^{2}+4 \lambda_{1} \mu_{2} x\left(\mu_{1}+x\right)
$$

and with :

$$
\sqrt{z}=\sqrt{p} e^{i \frac{\theta}{2}} \text { if } z=p e^{i \theta}, p \geq 0,-\pi<\theta \leq \pi
$$

The two branches give a two sheeted covering of the complex plane.

\section{$\underline{\text { Lemma } 1}$}

The algebraic function $Y(x)$ defined by $T(x, y)$ has two real branch points $\mathrm{x}^{* \star}, \mathrm{x}^{*}$ with $-\mu_{1}<\mathrm{x}^{* *}<\mathrm{x}^{*}<0$. 


\section{Proof}

From (14), the branch points of $Y(x)$ are the roots of $\Delta(x)$. Let $\mathrm{x}^{* *}$ and $\mathrm{x}^{*}$ these roots. It is easy to see that :

$$
-\mu_{1}<x^{\star \star} \underset{\underline{\operatorname{enf}}}{-\mu_{1}} \frac{\left(\sqrt{\lambda_{2}}+\sqrt{\mu_{2}}\right)^{2}}{\left(\sqrt{\lambda_{2}}+\sqrt{\mu_{2}}\right)^{2}+\lambda_{1}}<x^{\star \operatorname{def}}-\mu_{1} \frac{\left(\sqrt{\lambda_{2}}-\sqrt{\mu_{2}}\right)^{2}}{\left(\sqrt{\lambda_{2}}-\sqrt{\mu_{2}}\right)^{2}+\lambda_{1}}<0
$$

\section{Lemra 2}

For $\operatorname{Re} x^{\prime}=0$, the equation $T(x, y)=0$ has one $\operatorname{root} Y_{1}(x)$, in the right half-plane and one root $Y_{2}(x)$ in the left half-plane.

\section{Proof}

For $\operatorname{Re} x=0, T(x, y)=0 \Leftrightarrow y-\lambda_{1} \gamma_{1}(x) \cdot-\lambda_{2} \gamma_{2}(y)=0$.

It is well known (cf. $[\mathrm{COH} 69]$, p. 536) that, since $\beta_{2}()=.1-\gamma_{2}($. is not a lattice distribution, $y-\lambda_{1} \gamma_{1}(x)-\lambda_{2} \gamma_{2}(y)=0$ has exactly 2 zeros $Y_{1}(x)$ and $Y_{2}(x)$ with $\operatorname{Re} Y_{1}(x) \geq 0, \operatorname{Re} Y_{2}(x)<0$ if $\operatorname{Re} x=0, x \neq 0$. If $x=0, Y_{1}(x)=0$ and $Y_{2}(x)=\lambda_{2}-\mu_{2}<0$.

Let us denote $Y_{1}(x)$ the root of $T(x, y)$ such that $\operatorname{Re} Y_{1}(x) \geq 0$ if $\operatorname{Re} x=0$. We have :

$$
\mathrm{Y}_{1}(\mathrm{x})=\frac{\lambda_{1} \mathrm{x}-\left(\mu_{2}-\lambda_{2}\right)\left(\mu_{1}+\mathrm{x}\right)+\sqrt{\Delta(\mathrm{x})}}{2\left(\mu_{1}+\mathrm{x}\right)}
$$

$Y_{2}(x)$ will denote the other root.

From lemma 1 and (15), we see that the algebraic function defined by $Y_{1}(x)$ is analytic in the whole complex plane cut along $\left[x^{* *}, x^{*}\right]$.

\section{Lemma 3}

$\mathrm{Y}_{1}$ and $\mathrm{Y}_{2}$ map the cut $\left[\mathrm{x}^{\star *}, \mathrm{x}^{\star}\right]$ onto the circle $\mathcal{E}$ with centre $-\mu_{2}$ and radius $\sqrt{\lambda_{2} \mu_{2}}$. 


\section{Proof}

For $\mathrm{x} \in\left[\mathrm{x}^{\star *}, \mathrm{x}^{\star}\right], \Delta(\mathrm{x}) \leq 0$ and $\mathrm{Y}_{1}(\mathrm{x})$ and $\mathrm{Y}_{2}(\mathrm{x})$ are complex conjugate.

Let $a(x)$ and $b(x)$ be respectively the real part and the imaginary part of $\mathrm{Y}_{1}(\mathrm{x})$.

$$
\begin{gathered}
\text { If } x \in\left[x^{* *}, x^{*}\right] \text { then: } \\
Y_{1}(x)+Y_{2}(x)=\frac{\lambda_{1} x}{\mu_{1}+x}+\left(\lambda_{2}-\mu_{2}\right)=2 a(x) \\
Y_{1}(x) \cdot Y_{2}(x)=\frac{-\lambda_{1} \mu_{2} x}{\mu_{1}+x}=a^{2}(x)+b^{2}(x)
\end{gathered}
$$

It is readily seen that $a(x)$ and $b(x)$ are the real solutions of the equa-. tion $\left(a(x)+\mu_{2}\right)^{2}+b^{2}(x)=\lambda_{2} \mu_{2}$.

From the assumption $\rho_{1}+\rho_{2}<1$, we see that $-\mu_{2}+\sqrt{\lambda_{2} \mu_{2}}<0$. Hence $\mathscr{C}$ is entirely contained in the left half-plane $\{y \in \mathbb{a} / \operatorname{Re} y<0\}$.

Solving now the equation $T(x, y)=0$ in $x$ for fixed $y, y \in \mathbb{C}$, we find a unique solution :

$$
x(y) \stackrel{\operatorname{def}}{=} \mu_{1} \frac{y\left(\lambda_{2}-\mu_{2}-y\right)}{y^{2}+y\left(\mu_{2}-\lambda_{2}-\lambda_{1}\right)-\lambda_{1} \mu_{2}}
$$

$\mathrm{X}(\mathrm{y})$ has two real poles which are :

$$
\begin{aligned}
& y^{*} \stackrel{\operatorname{def}}{=} \frac{\lambda_{1}+\lambda_{2}-\mu_{2}+\sqrt{\left(\mu_{2}-\lambda_{2}-\lambda_{1}\right)^{2}+4 \lambda_{1} \mu_{2}}}{2}>0 \\
& y^{* *} \operatorname{def}_{=} \frac{\lambda_{1}+\lambda_{2}-\mu_{2}-\sqrt{\left(\mu_{2}-\lambda_{2}-\lambda_{1}\right)^{2}+4 \lambda_{1} \mu_{2}}}{2}<0
\end{aligned}
$$

It is easily seen that $-\mu_{2}<y^{* *}<-\mu_{2}+\sqrt{\lambda_{2} \mu_{2}}$. 


\section{Lemma 4}

For $\operatorname{Re} y=0$, the unique $\operatorname{root} x(y)$ of the equation $T(x, y)=0$ is located in the left half-plane.

\section{Proof}

For Re $y=0, T(x, y)=0 \Leftrightarrow \lambda_{1} \gamma_{1}(x)=y-\lambda_{2} \gamma_{2}(y)$.

Hence $\operatorname{Re} \lambda_{1} \gamma_{1}(x)=-\lambda_{2} \operatorname{Re} \gamma_{2}^{\prime}(y) \leq 0$.

The above inequality necessarily entails that $\operatorname{Re} x \leq 0$.

Lemma 5

$X\left(Y_{i}(x)\right)=x, \forall x \in \mathbb{C}, i=1,2$.

\section{$\underline{\text { Proof }}$}

Let $\mathrm{x} \in \mathbb{C}$. Then from the previous considerations the couples $\left(x, Y_{1}(x)\right),\left(x, Y_{2}(x)\right)$ are solutions of $T(x, y)=0$. In the same way, the couples $\left(X\left(Y_{1}(x)\right), Y_{1}(x)\right),\left(X\left(Y_{2}(x)\right), Y_{2}(X)\right)$ are also solutions. of $\mathrm{T}(\mathrm{x}, \mathrm{y})=0$.

Since $T(x, y)$ possesses a unique root for fixed. $y$, we necessarily have that $X\left(Y_{i}(x)\right)=x$, for $i=1,2$.

We introduce the intermediate function $\widetilde{B}$ defined by :

$$
\begin{aligned}
& \tilde{B}(y) \stackrel{\text { def }}{=} B(y) \cdot h(X(y), y) \text { for } \operatorname{Re} y \geq 0 . \\
& \text { 4.2. - Meromorphic continuation of } \tilde{B}(y)
\end{aligned}
$$

The domain within the contour $\mathscr{E}$ is called the interior domain and denoted as $\mathscr{E}^{+}$, whilst the complementary domain to $\mathcal{E}^{+}+\mathscr{E}$ is called the exterior domain and denoted by $\mathscr{L}^{-}$.

Let $D$ be defined as $\mathscr{D}^{\mathrm{def}} \mathscr{C}^{-} \mathrm{n}\{\mathrm{y} \in \mathbb{C} /$ Re y $<0\}$. In the following, Im will denote the imaginary axis of $\mathbb{C}$. 


\section{Lemma 6}

The algebraic function $X$ maps conformaliy $D$ on to the domain situated between the cut $\left[x^{* \star}, x^{\star}\right]$ and the curve $X(I m)$.

\section{Proof}

$X$ is a conformal mapping on $D$ since $X(y)$ is analytic for $y \in D$ and since the two roots $-\mu_{2} \pm \sqrt{\lambda_{2} \mu_{2}}$ of $\frac{\partial}{\partial y} x(y)$ do not belong to $D$.

The proof of $X(\mathscr{E})=\left[x^{* *}, x^{*}\right]$ is obviously obtained from lemma 3 and lemma 5 .

We can now continued $\widetilde{B}$ as a meromorphic function to $\mathscr{C}$. This is done in the following lemma.

\section{Lemma 7}

The function $\tilde{B}(y)$ which is meromorphic for Re $y \geq 0$ (it possesses a pole at infinity) can be continued as a meromorphic function to $\mathscr{G}^{-}$(it possesses also a pole at infinity for $\operatorname{Re} \mathrm{y}<0$ ).

\section{Proof}

From lemma 4, there exists a region $\Omega_{y}$ in the right half-plane; containing the imaginary axis and such that $\operatorname{Re} x(y) \leq 0, \nabla y \in \Re_{y}$.

$$
\begin{gathered}
\text { Hence, for } y \in \mathscr{R}_{\mathrm{y}}: \\
\mathrm{f}(\mathrm{X}(\mathrm{y}))+\mathrm{g}(\mathrm{X}(\mathrm{y}), \mathrm{y}) \mathrm{A}(\mathrm{X}(\mathrm{y}))+\widetilde{B}(\mathrm{y})=0
\end{gathered}
$$

Using lemma 6, equation (18) and the analycity of $A$ in the left half plane, we do a meromorphic continuation for $\widetilde{B}(y)$ for all y $\epsilon \varphi^{-}$.

At this point of the study, it is interesting to notice the following fact, in order to explain the introduction of the intermediate function $\widetilde{B}$. For the problems of the same type [FAY 79], [IAS 79], the unknown functions (here B) can usually be continued as meromorphic functions to the interior and/or the exterior domain of closed curves (here $\mathscr{C}$ ). But in this case, the known functions of the right hand of equation (11) cannot all be continued as meromorphic functions inside or outside the circle $\mathscr{G}$. 
Indeed, it is readily seen that $f(X(y))$ has an essential singular point for $\mathrm{y}=\mathrm{y}^{* *} \in \mathcal{E}^{+}$and that $\mathrm{h}(\mathrm{X}(\mathrm{y}), \mathrm{y})$ has two real branch points located in $]-\mu_{2}, O[$.

\section{3. - Reduction to a non-homogeneous Dirichlet problem on $C_{2}$}

We proceed as in [FAY, IAS 79].

$A(x)$ must be an analytic function for $\operatorname{Rex}<0$. In particular $A(x)$ is continuous on the cut $\left[x^{* *}, x^{*}\right]$ which yields :

$$
A^{+}(x)=A^{-}(x)
$$

for $x \in\left[x^{* *}, x^{*}\right]$ where $A^{+}(x)$ [resp. $\left.A^{-}(x)\right]$ denotes the limit of $A(x)$ from above [resp: below] the cut.

Since the algebraic functions $Y_{1}(x)$ and $Y_{2}(x)$ are complex conjugate for $x \in\left[x^{* *}, x^{*}\right]$, we obtain using equation (19)

$$
\tilde{B}(y) U(y)+v(y)=\tilde{B}(\bar{y}) U(\bar{y})+v(\bar{y}) \quad \text { for } y \in \mathscr{C}(20)
$$

where : $\quad U(y) \stackrel{\text { def }}{=} \cdot \frac{1}{g(x(y), y)}, \quad V(y) \stackrel{\text { def }}{=} \frac{f(x(y))}{g(x(y), y)}$

$\bar{y}$ denotes the complex conjugate of $y$.

Lemma 8.

$$
\begin{aligned}
& \text { Let } \hat{B}(y) \stackrel{\text { def }}{=} \tilde{\mathrm{B}}(y) U(y)-B_{1}(y) \text { where : } \\
& B_{1}(y) \stackrel{\operatorname{def}}{=} \sum_{j=1}^{2} \frac{r_{j}}{y-y_{j}}
\end{aligned}
$$

$\mathrm{y}_{1}, \mathrm{y}_{2}$ are the two distinct negative real roots of the polynomial

$$
\begin{array}{r}
\quad P(y) \stackrel{\text { def }}{=} y^{2}+y\left(\mu_{1}-\lambda_{1}+\mu_{2}-\lambda_{2}\right)+\mu_{1}\left(\mu_{2}-\lambda_{2}\right)+\mu_{2}\left(\mu_{1}-\lambda_{1}\right)-\mu_{1} \mu_{2}, \\
\quad r_{j}= \begin{cases}-\frac{f\left(x\left(y_{j}\right)\right)\left(y_{j}-y^{*}\right)\left(y_{j}-y^{* *}\right)}{y_{j} P^{\prime}\left(y_{j}\right)} & \text { if } y_{j} \in \mathscr{Y}+\bigcup \\
0 & \text { otherwise }\end{cases}
\end{array}
$$

and

where $P^{\prime \prime}(y)$ denotes the derivative of $P(y),(j=1,2)$. 
Then, $\hat{B}(y)$ is analytic in $\mathscr{C}^{-}$and continuous in $\mathscr{E}^{-}+\mathscr{C}$.

$\underline{\text { Proof }}$

$$
\text { Let } P(y) \stackrel{\text { def }}{=} y^{2}+y\left(\mu_{1}-\lambda_{1}+\mu_{2}-\lambda_{2}\right)+\mu_{1}\left(\mu_{2}-\lambda_{2}\right)+\mu_{2}\left(\mu_{1}-\lambda_{2}\right)-\mu_{1} \mu_{2} \text {. }
$$

From equations (8), (16), (21) we get

$$
U(y)=\frac{\left(y-y^{* *}\right)\left(y-y^{*}\right)}{y P(y)}
$$

We show in Appendix that the polynomial $P(y)$ always has two distinct negative real roots $y_{1}, y_{2}$. The location of these roots on the negative

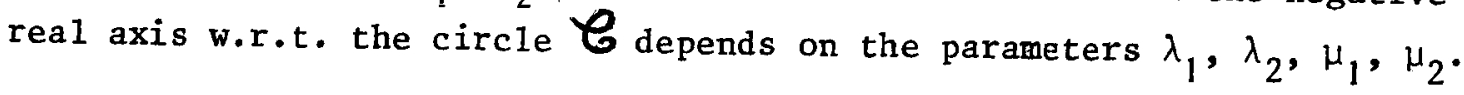

From lemma 7 , it is readily seen that $\frac{\tilde{B}(y)}{y}$ is analytic in $\varrho^{-}-(\tilde{B}(0)=0)-$ and continuous in $\boldsymbol{C}^{-}+\mathcal{C}$.

On the other hand, the rational function $\mathrm{y} \cdot \mathrm{U}(\mathrm{y})$ is analytic in $\mathscr{C}^{-}+\mathscr{C}$ except when $\mathrm{y}_{\mathrm{j}} \in \mathscr{D}+\mathscr{C}$, where it has a first order pole at this point, $(j=1,2)$.

Let $R_{j}$ be the residue of $y \cdot u(y)$ at $y=y_{j}$ if $y_{j} \in D+C$. Obviously, we have

$$
R_{j}=\frac{\left(y_{j}-y^{\star \star}\right)\left(y_{j}-y^{\star}\right)}{P^{\prime}\left(y_{j}\right)},(j=1,2) \text {. }
$$

Hence the function $\hat{B}(y)$ defined as

$$
\hat{B}(y) \stackrel{\text { def }}{=} \tilde{B}(y) U(y)-\sum_{j=1}^{2} \frac{r_{j}}{y-y_{j}} \text { with } r_{j}=\left\{\begin{array}{l}
\frac{\tilde{B}\left(y_{j}\right) R(j)}{y_{j}} \text { if } y_{j} \in \mathscr{D}+{ }^{\prime} \\
0 \quad \text { otherwise }
\end{array}\right.
$$

is clearly analytic in $\mathscr{C}^{-}$and continuous in $\mathscr{C}^{-}+\mathscr{C},(j=1,2)$.

Finally, it is seen from equation (18) that $\tilde{B}\left(y_{j}\right)=-f\left(X\left(y_{j}\right)\right)$.

Using lemma 8 and equation (20) we get :

$$
\hat{B}(y)+\hat{v}(y)=\hat{B}(\bar{y})+\hat{v}(\bar{y}) \text { for } y \in C_{0}
$$

where : $\quad \hat{\mathrm{v}}(\mathrm{y}) \stackrel{\text { def }}{=} \mathrm{v}(\mathrm{y})+\mathrm{B}_{1}(\mathrm{y})$.

We notice that $\hat{v}(y)$ is continuous on $\zeta$, even if $y_{j} \in \zeta_{\text {, since }} r_{j}$ is also the residue of $-V(y)$ at the point $y_{j}$ for $y_{j} \in \zeta_{,}(j=1,2)$. 
The coefficient of the known function $\hat{v}(y)$ being real, we may rewrite equation (22) as :

$$
\operatorname{Re}(-i \hat{B}(y))=\Psi(y) \quad \text { for } y \in \ell
$$

where : $\quad \Psi(y) \stackrel{\text { def }}{=}-\operatorname{Im} \hat{\mathrm{V}}(\mathrm{y})$.

Im $z$ is the imaginary part of the complex number $z$. The problem is now reduced to the following : find a function $\hat{\mathbf{B}}$ analytic in $\zeta^{-}$, continuous in $\varrho^{-}+\mathscr{C}$, satisfying the boundary condition (23), where $\Psi(y)$ is a known function, 'continuous on $\zeta$.

This is a non-homogeneous Dirichlet problem for the circle $\varphi$. The solution is given by : [GAH 66], [MUS 46]

$$
\hat{B}(y)=-\frac{1}{2 \pi} \quad \int_{C} \Psi(\omega(t)) \frac{t+\omega^{-1}(y)}{t-\omega^{-1}(y)} \frac{d t}{t}+D, \quad y \in b^{-}
$$

where $D$ is a real constant, and $\omega(y) \stackrel{\text { def }}{=} \sqrt{\lambda_{2} \mu_{2}} y-\mu_{2}$ maps conformally $\zeta^{-}$ onto the domain outside the unit circle $C$.

The constant $D$ will be determined in the next section.

\section{4. - Workload distribution}

From lemma 8 we get :

$$
B(y)=\frac{y-X(y)}{X(y)-Z(y)}\left(\hat{B}(y)+B_{1}(y)\right) \text { for Re } y \geq 0
$$

We must verify that $x(y)-z(y) \neq 0$ for Re $y \geq 0, y \neq 0$. Let us assume that $x(y)=Z(y)$. From the definitions of $x$ and $z$ this implies that $X(y)=y$ or equivalently y. $P(y)=0$. Appendix $A$ shows us that $P$ only vanishes in the left half plane. Hence $x(y)-Z(y) \neq 0$ for $\operatorname{Re} y \geq 0, y \neq 0$ and $B(y)$ given by equation (25) is analytic for Re $y>0$. 
This enable us to determine $A(x)$ for $\operatorname{Re} x=0$.

From Lemma 2, we know that $\operatorname{Re} Y_{1}(x) \geq 0$ for $\operatorname{Re} X=0$, and that $Y_{1}(x)$ is analytic in the whole complex plane cut along $\left[x^{* *}, x^{*}\right]$.

Hence, using equation (26) and lemma 5 , we find :

$$
A(x)=-\frac{f(x)}{Y_{1}(x)-x}-\hat{B}\left(Y_{1}(x)\right)-B_{1}\left(Y_{1}(x)\right) \text { for } \operatorname{Re} x=0
$$

The continuity of $A(x)$ for $\operatorname{Re} x=0$ follows from the fact that $Y_{1}(x)-x$ has no root for $\operatorname{Re} x=0, x \neq 0$. Indeed, let us assume that $Y_{1}(x)=x$. This implies that $x \cdot P(x)=0$. We again conclude using Appendix A.

It remains to compute the constant $D$ of equation (24). It is easily seen that $Y_{2}(x)$ given by

$$
Y_{2}(x)=\frac{\lambda_{1} x-\left(\mu_{2}-\lambda_{2}\right)\left(\mu_{1}+x\right)-\sqrt{\Delta(x)}}{2\left(\mu_{1}+x\right)} \quad \text { (see section 4.1) }
$$

is positive for $x \in]-\infty,-\mu_{1}[$.

So, for $x \in]-\infty,-\mu_{1}[$ we have :

$$
A(x)=-\frac{f(x)}{Y_{2}(x)-x}-\hat{B}\left(Y_{2}(x)\right)-B_{1}\left(Y_{2}(x)\right)
$$

On the other hand, $\lim A(x)=0 . \quad$ (see equations (8))

$$
\begin{aligned}
& \mathbf{X} \rightarrow-\infty \\
& \mathbf{x} \in \mathbf{R}
\end{aligned}
$$

Hence, from equation (27),

$$
\hat{\mathrm{B}}\left(\mathrm{y}^{*}\right)+\mathrm{B}_{1}\left(\mathrm{y}^{*}\right)=0
$$

since $\lim _{\substack{x \rightarrow-\infty \\ x \in \mathbf{R}}} Y_{2}(x)=y^{*}$.

Fina11y, we obtain from equations (24) and (28):

$$
D=\frac{1}{2 \pi} \int_{C} \Psi(\omega(t)) \frac{t+\omega^{-1}\left(y^{\star}\right)}{t-\omega^{-1}\left(y^{*}\right)} \frac{d t}{t}-\sum_{j=1}^{2} \frac{r_{j}}{y^{\star}-y_{j}}
$$


The results of this study are summarized in the following theorem.

\section{Theorem}

We have for $\operatorname{Re} x=0, \operatorname{Re} y: \geq 0$

$$
\begin{aligned}
H(x, y) & =e^{-x c}\left\{\frac{\left(1-\rho_{1}-\rho_{2}\right) x e^{x c}+(y-x) A(x)+(x-z(y)) B(y) /\left(\mu_{1}+x\right)}{y-\lambda_{1} \gamma_{1}(x)-\lambda_{2} \gamma_{2}(y)}\right. \\
& \left.-\frac{(x-z(y)) B(y)}{\left(\mu_{1}+x\right)\left(x-\lambda_{1} \gamma_{1}(x)-\lambda_{2} \gamma_{2}(y)\right)}\right\} ;
\end{aligned}
$$

where $A(x)$ and $B(y)$ are respectively given in equations (26) and (25).

In Appendix B we give reduced formulas for the computation of the mean workload in each queue. Figures 1 and 2 show the effect of the control parameter $c$ upon the mean workload in each queue, for two given traffic intensity (weak and heavy) levels.

I should like to thank G. Fayolle for suggesting this research topic and for many helpful discussions during the course of this work, and also to express my appreciation to $F$. Baccelli and Ph. Robert for their help. 


\section{APPENDIX A}

\section{$\underline{\text { Lemma }}$}

$$
P(y) \stackrel{\text { def }}{=} y^{2}+y\left(\mu_{1}-\lambda_{1}+\mu_{2}-\lambda_{2}\right)+\mu_{1}\left(\mu_{2}-\lambda_{2}\right)+\mu_{2}\left(\mu_{1}-\lambda_{1}\right)-\mu_{1} \mu_{2}
$$

always has two distinct negative real roots. Moreover, the position of these roots on the negative real axis with respect to the circle $\mathcal{E}$, depends on the values of the parameters $\lambda_{1}, \lambda_{2}, \mu_{1}, \mu_{2}$.

\section{Proof}

We assume that $\rho_{1}+\rho_{2}<1$ and $\lambda_{1} \neq 0, \lambda_{2} \neq 0$.

Let us define the polynomial $T(z)$ as :

$$
T(z) \stackrel{\text { def }}{=} z^{2}+z\left(\alpha^{2}\left(1-\rho_{1}\right)+1-\rho_{2}\right)+\alpha^{2}\left(1-\rho_{1}-\rho_{2}\right)
$$

where :

$$
\alpha \stackrel{\operatorname{def}}{=} \sqrt{\frac{\mu_{1}}{\mu_{2}}} \neq 0 .
$$

We have :

$$
P(y)=\mu_{2}^{2} T\left(\frac{y}{\mu_{2}}\right) .
$$

The discriminant of the polynomial $\mathrm{T}$ is :

$$
\begin{aligned}
& \Delta_{T}(\alpha)=\left(\alpha^{2}\left(1-\rho_{1}\right)+1-\rho_{2}\right)^{2}-4 \alpha^{2}\left(1-\rho_{1}-\rho_{2}\right) \\
&=\left(\alpha^{2}\left(1-\rho_{1}\right)+\left(1-\rho_{2}\right)+2 \alpha \sqrt{1-\rho_{1}-\rho_{2}}\right)\left(\alpha^{2}\left(1-\rho_{1}\right)+\left(1-\rho_{2}\right)\right. \\
&-2 \alpha \sqrt{\left.1-\rho_{1}-\rho_{2}\right)}
\end{aligned}
$$

Hence :

$$
\operatorname{sgn}\left(\Delta_{T}(\alpha)\right)=\operatorname{sgn}\left(\alpha^{2}\left(1-\rho_{1}\right)+\left(1-\rho_{2}\right)-2 \alpha \sqrt{1-\rho_{1}-\rho_{2}}\right) .
$$


Since the discriminant of this polynomial in $\alpha$ is always strictly negative, it follows that $\Delta_{T}(\alpha)>0$.

The easiest way to prove the second part of the lemma is to choose particular values for $\rho_{1}, \rho_{2}, \alpha$.

First, let us examine the case where $\rho_{1}=\rho_{2}=\rho$ and $\alpha=1$. Then the roots of $T$ are $z_{1}=-1$ and $z_{2}=-1+2 \rho$. These roots must be positioned with respect to the interval $I_{p} \operatorname{def}_{=}[-1-\sqrt{p} ;-1+\sqrt{\rho}]$.

- if $\rho<\frac{1}{4}$ then $z_{1}, z_{2} \in I_{\rho} \quad\left(\left(z_{1}, z_{2}\right) \in \varphi^{+}\right)$.

- if $\rho=\frac{1}{4}$ then $z_{1}, z_{2} \in I_{1 / 4}$ and $z_{2}=-\frac{1}{2}\left(z_{1} \in \mathscr{C}^{+}, z_{2} \in \zeta\right)$.

- if $\rho>-\frac{1}{4}$ then $z_{1} \in I_{\rho}$ and $z_{2} \notin I_{\rho}\left(z_{1} \in \mathcal{C}^{+}, z_{2} \in \mathcal{C}^{-}\right)$.

The case-remains where $z_{1}$ and $z_{2}$ do not belong to $I_{\rho_{2}}$. This happens, in particular, if $\rho_{1}=\frac{7}{10}, \rho_{2}=\frac{1}{4}$ and $\alpha=3$. Then $\left(z_{1}, z_{2}\right) \in \varphi^{-}$. 


\section{APPENDIX B}

From the theorem of section 4.4 we have :

$$
\begin{aligned}
& E\left\{V_{1}\right\}=-\left.\frac{\partial}{\partial x} H(x, 0)\right|_{x=0} \\
& E\left\{V_{2}\right\}=-\left.\frac{\partial}{\partial y} H(0, y)\right|_{y=0}
\end{aligned}
$$

A tedious computation, involving first and second order expansions of $\mathrm{X}, \mathrm{Y}_{1}, \mathrm{Z}$ as well as their derivatives, yields :

$$
\begin{aligned}
E\left\{V_{1}\right\}= & -\frac{1}{\lambda_{1}}\left(A(0)\left(1-\mu_{1} c\right)+\mu_{1} A^{\prime}(0)+c B(0)-\left(1-\rho_{1}-\rho_{2}\right)\right) \\
& -\left(\frac{1+\left(\mu_{1}-\lambda_{1}\right) c}{\left(\mu_{1}-\lambda_{1}\right)^{2}}\right) B(0) \\
E\left\{V_{2}\right\}= & \frac{\rho_{2}}{\mu_{2}\left(1-\rho_{2}\right)^{2}} A(0)+\frac{1-2 \rho_{2}}{\lambda_{2} \mu_{1}\left(1-\rho_{2}\right)^{2}} B(0) Z^{\prime}(0) \\
& +\frac{1}{\mu_{1} \rho_{2}\left(1-\rho_{2}\right)}\left(\frac{B(0) Z^{\prime \prime}(0)}{2}+B^{\prime}(0) Z^{\prime}(0)\right)
\end{aligned}
$$

where :

$$
\begin{aligned}
& Y_{1}^{\prime}(0)=\frac{\rho_{1}}{I-\rho_{2}}, \\
& Z^{\prime}(0)=\frac{\rho_{2}}{1-\rho_{1}}, \\
& X^{\prime}(0)=\frac{1-\rho_{2}}{\rho_{1}},
\end{aligned}
$$




$$
\begin{aligned}
& \mathrm{Y}_{1}^{\prime \prime}(0)=\frac{\left(\mu_{2}-\lambda_{2}\right)^{2}-3 \lambda_{1} \mu_{2}+\lambda_{1} \lambda_{2}}{2\left(\mu_{2}-\lambda_{2}\right) \mu_{1}^{2}}+\mathbf{b} \\
& \mathbf{b}=\frac{1}{2 \mu_{1}^{2}\left(\mu_{2}-\lambda_{2}\right)}\left(\left(\lambda_{1}+\lambda_{2}-\mu_{2}\right)^{2}+4 \lambda_{1} \mu_{2}\right. \\
&-\frac{\left.\left(\left(\mu_{2}-\lambda_{2}\right)^{2}+\lambda_{1}\left(\lambda_{2}+\mu_{2}\right)\right)\left(2\left(\mu_{2}-\lambda_{2}\right)^{2}+\lambda_{1}\left(\lambda_{2}+\mu_{2}\right)\right)\right)}{\left(\mu_{2}-\lambda_{2}\right)^{2}}
\end{aligned}
$$

$Z^{\prime \prime}(0)=$ the same expression as $\mathrm{Y}_{1}^{\prime \prime}(0)$, changing respectively $\lambda_{1}, \lambda_{2}, \mu_{1}, \mu_{2}$ for $\lambda_{2}, \lambda_{1}, \mu_{2}, \mu_{1}$;

$x^{\prime \prime}(0)=2 \mu_{1} \frac{\left(\mu_{2}-\lambda_{2}\right)^{2}+\lambda_{1} \lambda_{2}}{\lambda_{1}^{2} \mu_{2}^{2}}$,

$A(0)=\frac{B(0)}{\mu_{1}-\lambda_{1}}+1-\rho_{2} \quad, \quad($ from equation $(26))$

$$
B(0)=-\mu_{1}\left(1-p_{1}\right) \Phi(0), \quad \text { (from equation (25)) }
$$

$$
\begin{aligned}
B^{\prime}(0)= & \mu_{1} \Phi(0)\left(\frac{Z^{\prime \prime}(0)\left(1-X^{\prime}(0)\right)-X^{\prime \prime}(0)\left(1-Z^{\prime}(0)\right)}{2\left(X^{\prime}(0)-Z^{\prime}(0)\right)^{2}}\right) \\
& +\frac{\left[X^{\prime}(0) \Phi(0)+\mu_{1} \Phi^{\prime}(0)\right]\left(1-X^{\prime}(0)\right)}{X^{\prime}(0)-Z^{\prime}(0)}
\end{aligned}
$$

$\Phi(y) \stackrel{\operatorname{def}}{=} \hat{B}(y)+B_{1}(y)$,

$\Phi(0)=\frac{1}{2 \pi} \int_{C} \Psi(\omega(t))\left(\frac{t+\omega^{-1}\left(y^{*}\right)}{t-\omega^{-1}\left(y^{*}\right)}-\frac{t+\omega^{-1}(0)}{t-\omega^{-1}(0)}\right) \frac{d t}{t}-\sum_{j=1}^{2} \frac{r_{j}}{y^{*}-y_{j}}-\sum_{j=1}^{2} \frac{r_{j}}{y_{j}}$,

$\Phi^{\prime}(0)=-\frac{\sqrt{\lambda_{2} \mu_{2}}}{\pi} \int_{C} \frac{\Psi(\omega(t))}{\left(t \sqrt{\lambda_{2} \mu_{2}}-\mu_{2}\right)^{2}} d t-\sum_{j=1}^{2} \frac{r_{j}}{y_{j}^{2}}$,

$A^{\prime}(0)=\left(\frac{1-\rho_{1}-\rho_{2}}{1-Y_{1}^{\prime}(0)}\right)\left(\frac{Y_{1}^{\prime \prime}(0)}{2\left(1-Y_{1}^{\prime}(0)\right)}+c\right)-Y_{1}^{\prime}(0) \Phi^{\prime}(0):$ 
REFERENCES

[BLA 82] BLANC, J.P.C. : Application of the theory of boundary value problems in the analysis of a queueing model with paired services. Thesis, University of Utrecht, Utrecht (1982).

[BAC, FAY 82] BACCELLI, F. and FAYOLLE, G. : Two dimensional diffusion processes with boundary and jumps. Application to coupled queues. Rapport de Recherche INRIA $\mathrm{n}^{\circ} 160$, (1982).

[BOX, COH 81] BOXMA, O.J. and COHEN, J.W. : The M/G/l queue with alternating service formulated as a Riemann-Hilbert problem. Performance 81 , F.J. Kylstra Ed. North Holland Publishing Company, (1981).

[COH 69] COHEN, J.W. : The single server. Amsterdam, North Holland Publishing, (1969).

[COH 79] COHEN, J.W. : On the M/G/2 queueing mode1. Stoch. Proc. Appl. 12, 3, (1982).

[FAY 79] FAYOLLE, G. : Méthodes analytiques pour les files d'attente couplées. Thèse, Université Paris VI, (1979).

[FAY, IAS 79] FAYOLLE, G. and IASNOGORODSKI, R. : Two coupled processors : the reduction to a Riemann-Hilbert problem. $Z$. Wahrscheinlichkeitsth 47, 325-351, (1979).

[FAY, KIN, MIT 82] FAYOLLE, G. and KING, P.J.B. and MITRANI, I. : The solution of certain two-dimensional Markov models. Adv. App1. Prob. 14, 295-308, (1982).

[GHA 66] GHAKOV, F.D. : Boundary value problems. Pergamon Press, Oxford, (1966).

[IAS 79] IASNOGORODSKI, R. : Problèmes-frontières dans les files d'attente. Thèse, Université Paris VI, (1979).

[KLE 75] KLEINROCK, L. : Queueing systems. Vo1. 1, Academic Press, (1975).

[MIK 81] MIKOU, N. : Modèles de réseaux de files d'attente avec pannes. Thèse, Université Paris XI, Orsay, (1981).

[MUS 46] MUSKHELISHVILI, N.I.: Singular integral equations. Groningen, Holland-Moscow, P. Noordhoff, (1946).

[SMI 72] SMIRNOV, V. : Cours de Mathématiques Supérieures. Tome 3, Part. 2, Edition de Moscou, (1972).

[TAK 52]

TAKÁCS, L.: Introduction to the theory of queues. Oxford Univ. Press, (1952). 


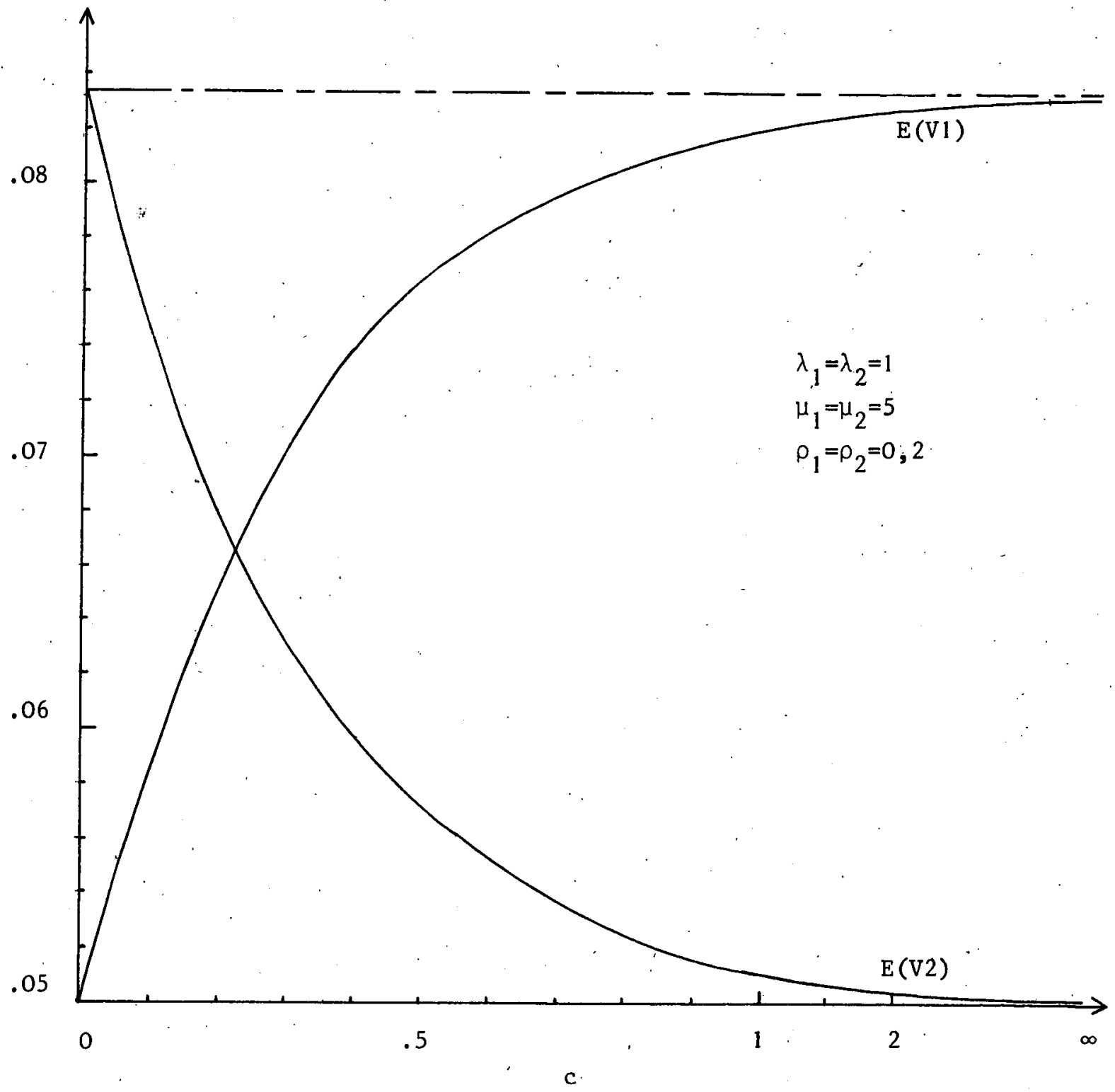

Figure 1: mean workoad in each queue for a weak traffic intensity 


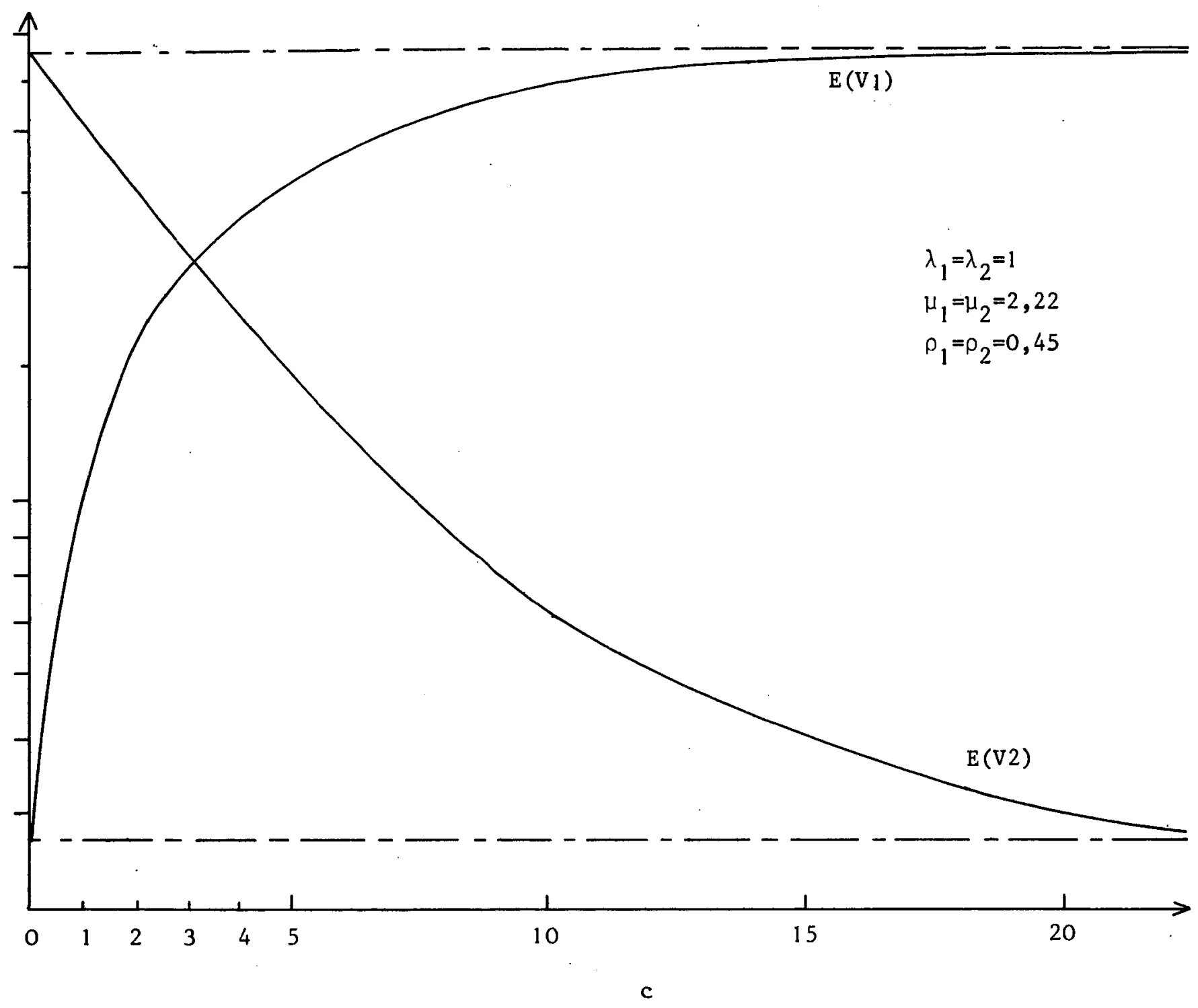

Figure 2 : mean workload in each queue for a heavy traffic intensity. 
